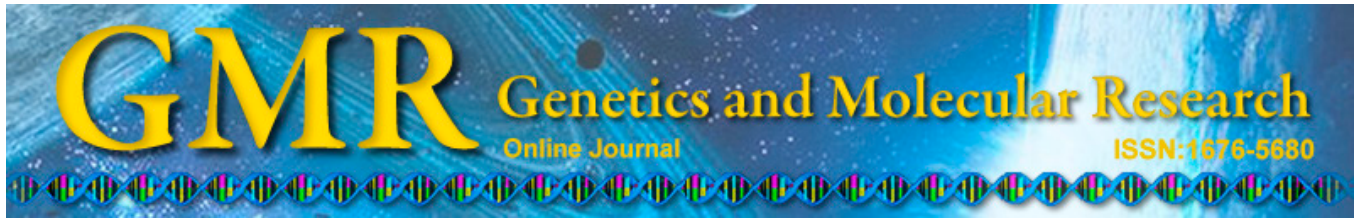

\title{
Expression and significance of S100P, CD147, and OCT4 in different prostate cancer tissue TNM stages
}

\author{
Q. Wang ${ }^{1}$, J.G. Zhang ${ }^{1}$ and W. Wang ${ }^{2}$ \\ ${ }^{1}$ Department of Urology, \\ The First Affiliated Hospital of Henan University of Science and Technology, \\ Henan, China \\ ${ }^{2}$ Department of Radiological, \\ Nanyang Hospital of Traditional Chinese Medicine, Henan, China \\ Corresponding author: J.G. Zhang \\ E-mail: jgzhang929@126.com
}

Genet. Mol. Res. 14 (2): 6844-6851 (2015)

Received September 18, 2014

Accepted February 11, 2015

Published June 18, 2015

DOI http://dx.doi.org/10.4238/2015.June.18.27

\begin{abstract}
The aim of this project was to investigate the expression and significance of S100P, CD147, and OCT4 in prostate cancer tissue at different TNM stages. We enrolled 54 patients with prostate cancer, 40 with benign prostatic hyperplasia, and 20 subjects with normal prostates. S100P, CD147, and OCT4 were detected by immunohistochemistry. The positive rate of S100P detection was $18.52 \%$ in prostate cancer tissues, significantly lower than in normal and benign prostate hyperplasia tissues $(\mathrm{P}<0.05)$. The positive expression rate of CD147 and OCT4 were 100 and $77.38 \%$ in prostate cancer tissue, respectively, both markedly higher than in normal and benign prostate hyperplasia tissue $(\mathrm{P}<0.05)$. The positive rate of $\mathrm{S} 100 \mathrm{P}$ in stage $\mathrm{V}$ was 0 , which was significantly lower than in stages I $(37.50 \%)$ and II $(35.71 \%)(\mathrm{P}<0.05)$. OCT4 expression in stages III (86.67\%) and V (94.12\%) was higher than in stage I $(37.50 \%)$. The positive rate of S100P in patients with distant metastasis was $4 \%$, which was significantly lower than that in patients without metastases
\end{abstract}


$(\mathrm{P}<0.05)$. In contrast, the positive rate of OCT4 in patients with distant metastasis was $92 \%$. S100P, CD147, and OCT4 expression in prostate cancer patients with different degrees of differentiation had no significant difference $(\mathrm{P}>0.05)$. Overall, our results demonstrated that S100P expression in prostate cancer tissue was significantly decreased, whereas CD147 and OCT4 expression was increased. Their expression levels were closely associated with TNM stage and distant metastasis, but were not related to the degree of differentiation.

Key words: Prostate cancer; TNM grading; S100P; CD147; OCT4

\section{INTRODUCTION}

Prostate cancer is one of the most common malignant tumors and its incidence has shown a trend of yearly increase following men's eating and lifestyle changes. In recent years, with the rapid development of immunology and molecular biology techniques, tumor markers have played an important role in the differential diagnosis of malignant tumors, prognosis evaluation, and follow-up (Javanmard et al., 2014). The survival rate and treatment for patients with prostate cancer are closely related to tumor stage. Early detection and early treatment can help improve patient prognosis (Vasiljevic et al., 2014). The present study analyzed the positive rate of the markers S100P, CD147, and OCT4 in prostate cancer tissues with different TNM staging to provide a reference for clinical diagnosis and treatment.

\section{MATERIAL AND METHODS}

\section{General information}

The protocol of this study was approved by The First Affiliated Hospital of Henan University of Science and Technology (Henan, China). Informed consents were obtained from all subjects. We enrolled 54 patients with prostate cancer, 40 patients with prostatic hyperplasia, and 20 subjects with normal prostate tissue between April 2012 and January 2014 from the First Affiliated Hospital of Henan University of Science and Technology Department of Urology. All diagnoses were confirmed by pathology. Inclusion criteria were: 1) tissue was sampled prior to radiotherapy, chemotherapy, or hormone therapy; 2) cases contained complete clinical staging, pathological grading, and other general information; and 3) diagnoses were confirmed by postoperative pathology. Exclusion criteria were: 1) cancer was combined with malignant tumors of other organs; or 2) case information was incomplete. The mean age of the 54 patients with prostate cancer was 58.1 \pm 3.9 years, and ranged from 37-77 years. TNM staging according to the American Joint Committee on Cancer (Tong et al., 2014) was: 8 patients in stage I, 14 in stage II, 15 in stage III, and 17 in stage V. Furthermore, 13 patients exhibited distant metastasis, and 41 had no metastasis. Degree of differentiation according to the Gleason score standard (Jones, 2014) was: 21 patients were poorly differentiated, 24 patients were moderately differentiated, and 9 patients had well differentiated tumors. The mean age of the 40 patients with prostate hyperplasia was $57.8 \pm 4.3$ years, and ranged from 35-79 years. The average age of the 20 subjects with healthy physical examination was $56.9 \pm 5.1$ years and ranged between $30-75$ years. 


\section{Methods}

\section{Materials}

The SP immunohistochemical kit and chromogenic reagent kit were purchased from GIBCO (Gaithersburg, MD, USA). Primary antibodies for CD147, S100P, and OCT4 were bought from Baygene Biotech, Beijing, China.

\section{Detection method}

The tissues were stained with routine $\mathrm{H} \& \mathrm{E}$ and relative immunohistochemistry was performed according to the kit manuals.

\section{Positive judgment}

A section positive for prostate cancer was used a positive control, while phosphate buffered saline instead of antibody was treated as a negative control. Five different visual fields were randomly selected from each section for counting. Dyeing result scores were taken from the positive cell percentage and staining intensity. Positive cell percentage was determined according to the proportion of positive cells among the normal cells and evaluated as follows: 0 points, positive staining cells less than $5 \%$; 1 point, positive staining cells between 5 and $25 \% ; 2$ points, positive staining cells between 26 and 50\%; 3 points, positive staining cells more than $50 \%$. Cell staining intensity criteria were: 0 points, no coloring; 1 point, moderate coloring; 2 points, high coloring. The total score was the product of the positive cell percentage and the staining intensity: negative, $0-1$ points; weakly positive, 2-3 points; moderately positive, 3-4 points; and 5-6 points, strongly positive.

\section{Statistical analysis}

Different groups were tested by ANOVA, while the significant differences were analyzed by the Bonferroni test. Enumeration data were analyzed using the chi-square test. All statistical analyses were performed using the SPSS20.0 software (SPSS, Chicago, IL, USA). $P$ values $<0.05$ were considered to be statistically significant.

\section{RESULTS}

\section{Comparison of S100P, CD147, and OCT4 among different tissues}

The positive rate of S100P expression was $18.52 \%$ in prostate cancer tissues, which was significantly lower than that in normal and benign prostate hyperplasia tissues $(\mathrm{P}<0.05)$. The positive expression rate of CD147 and OCT4 were 100 and $77.38 \%$ in prostate cancer tissue, respectively, and they were both markedly higher than in normal and benign prostate hyperplasia tissues $(\mathrm{P}<0.05)$ (Table 1). Positive $\mathrm{S} 100 \mathrm{P}$ presented claybank granules primarily concentrated in cancer cell cytoplasm with few expressed in the nucleus (Figure 1A); positive CD147 presented claybank granules that were primarily concentrated in the nucleus with 
fewer expressed in the cytoplasm (Figure 1B); positive OCT4 presented brown granules and had similar distribution as CD147 (Figure 1C).

Table 1. Comparison of S100P, CD147, and OCT4 expression status among different tissues [N (\%)].

\begin{tabular}{|c|c|c|c|c|c|c|c|}
\hline \multirow[t]{2}{*}{ Group } & \multirow[t]{2}{*}{ Number } & \multicolumn{2}{|c|}{ S100P } & \multicolumn{2}{|c|}{ CD147 } & \multicolumn{2}{|c|}{ OCT4 } \\
\hline & & Positive & Negative & Positive & Negative & Positive & Negative \\
\hline Normal & 20 & $19(95.00)$ & $1(5.00)$ & $2(10.00)$ & $18(90.00)$ & $1(5.00)$ & $19(95.00)$ \\
\hline Benign prostatic hyperplasia & 40 & $36(90.00)$ & $4(10.00)$ & $7(17.50)$ & $33(82.50)$ & $9(22.50)$ & $31(77.50)$ \\
\hline Prostate cancer & 54 & $10(18.52)^{*}$ & $44(81.48)$ & $54(100.00)^{*}$ & 0 & $42(77.38)^{*}$ & $12(22.22)$ \\
\hline
\end{tabular}

$* \mathrm{P}<0.05$ compared with normal and benign prostatic hyperplasia.
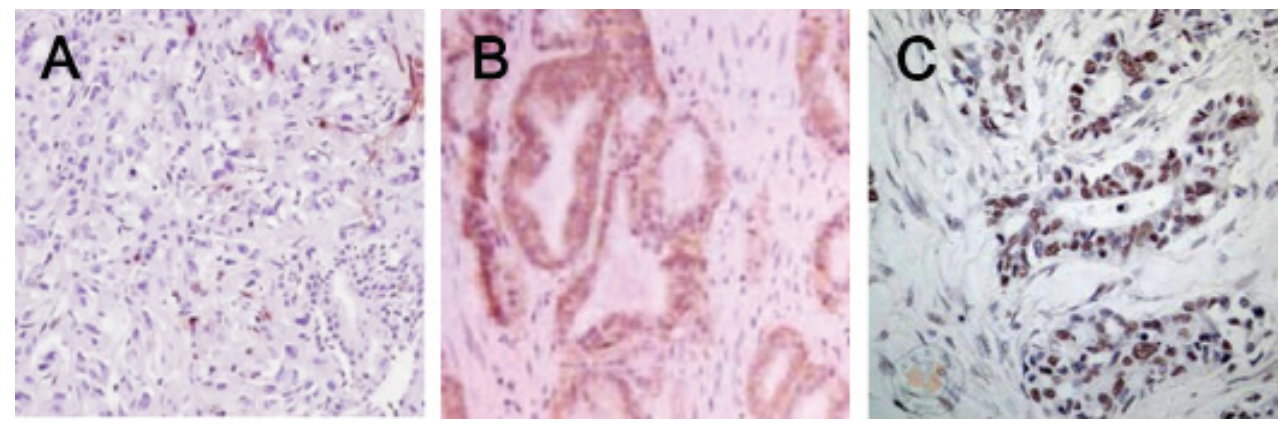

Figure 1. Positive expression of S100P, CD147, and OCT4 in prostate cancer by immunohistochemistry. A. Positive expression of S100P. B. Positive expression of CD147. C. Positive expression of OCT4.

\section{Comparison of S100P, CD147, and OCT4 in prostate cancer among different TNM stages}

The positive rate of $\mathrm{S} 100 \mathrm{P}$ in stage $\mathrm{V}$ was 0 , which was significantly lower than in stages I $(37.50 \%)$ and II $(35.71 \%)(\mathrm{P}<0.05)$. CD147 positive rates in all stages were $100.00 \%$, with a strong positive rate of $57.41 \%$ that was significantly higher than the percentage of weakly and moderately CD147-positive staining $(\mathrm{P}<0.05)$. OCT4 expression in stages III $(86.67 \%)$ and V (94.12\%) was higher than that in stage I (37.50\%) (Table 2).

Table 2. Comparison of S100P, CD147, and OCT4 expression status in prostate cancer among different TNM stages.

\begin{tabular}{|c|c|c|c|c|c|c|c|c|c|c|c|c|c|}
\hline \multirow[t]{2}{*}{ TNM stage } & \multirow[t]{2}{*}{ Number } & \multicolumn{4}{|c|}{ S100P } & \multicolumn{4}{|c|}{ CD147 } & \multicolumn{4}{|c|}{ OCT4 } \\
\hline & & - & + & ++ & +++ & - & + & ++ & +++ & - & + & ++ & +++ \\
\hline Stage I & 8 & 5 & 3 & 0 & 0 & 0 & 2 & 3 & 3 & 5 & 1 & 1 & 1 \\
\hline Stage II & 14 & 9 & 4 & 1 & 0 & 0 & 3 & 4 & 7 & 4 & 2 & 3 & 5 \\
\hline Stage III & 15 & 13 & 2 & 0 & 0 & 0 & 2 & 3 & 10 & 2 & 3 & 4 & 6 \\
\hline Stage V & 17 & 17 & 0 & 0 & 0 & 0 & 2 & 4 & 11 & 1 & 3 & 4 & 9 \\
\hline Total & 54 & 44 & 9 & 1 & 0 & 0 & 9 & 14 & 31 & 12 & 9 & 12 & 21 \\
\hline
\end{tabular}

$\mathrm{TNM}=$ tumor, node, metastasis. 


\section{Comparison of S100P, CD147, and OCT4 in prostate cancer with distant metastasis}

The positive rate of S100P in patients with distant metastasis was $4 \%$, which was significantly lower than that of patients without metastases $(\mathrm{P}<0.05)$. In contrast, the positive rate of OCT4 in patients with distant metastasis was $92 \%$, demonstrating the opposite tendency to that of S100P (Table 3).

\begin{tabular}{|c|c|c|c|c|c|c|c|}
\hline \multirow[t]{2}{*}{ Distant metastasis } & \multirow[t]{2}{*}{ Number } & \multicolumn{2}{|c|}{ S100P } & \multicolumn{2}{|c|}{ CD147 } & \multicolumn{2}{|c|}{ OCT4 } \\
\hline & & Positive & Negative & Positive & Negative & Positive & Negative \\
\hline With & 25 & $1(4.00)$ & $24(96.00)$ & $25(100.00)$ & 0 & $23(92.00)$ & $2(8.00)$ \\
\hline Without & 29 & $9(31.03)$ & $20(68.97)$ & $29(100.00)$ & 0 & $19(65.52)$ & $10(34.48)$ \\
\hline
\end{tabular}

\section{Comparison of S100P, CD147, and OCT4 in prostate cancer with different differentiation}

S100P, CD147, and OCT4 expression in prostate cancer patients with different degrees of differentiation had no significant difference $(\mathrm{P}>0.05)$ (Table 4$)$.

\begin{tabular}{|c|c|c|c|c|c|c|c|}
\hline \multirow[t]{2}{*}{ Differentiation } & \multirow[t]{2}{*}{ Number } & \multicolumn{2}{|c|}{ S100P } & \multicolumn{2}{|c|}{ CD147 } & \multicolumn{2}{|c|}{ OCT4 } \\
\hline & & Positive & $\overline{\text { Negative }}$ & Positive & Negative & Positive & Negative \\
\hline Poor & 21 & $4(19.05)$ & $17(80.95)$ & $21(100.00)$ & 0 & $17(80.95)$ & $4(19.05)$ \\
\hline Moderate & 24 & $4(16.67)$ & $20(83.33)$ & $24(100.00)$ & 0 & $19(79.17)$ & $5(20.83)$ \\
\hline Well & 9 & $2(22.22)$ & $7(77.78)$ & $9(100.00)$ & 0 & $6(66.67)$ & $3(33.33)$ \\
\hline
\end{tabular}

\section{DISCUSSION}

The incidence of prostate cancer has been increasing year by year. Comprehensive treatment with radical prostatectomy and radioactive particle implantation has had significant effect, especially on improving the prognosis of early stage patients. However, clinical studies (Monma et al., 2013) have revealed that $17-51 \%$ of prostate cancer appeared as distant metastasis, while local recurrence rates were 6-21\%. Relapses usually appeared within three years after surgery, with a few patients showing postoperative recurrence after five years. The one-year survival rate of patients with metastasis and recurrence is quite low, and the treatment is more difficult. Clinical research has shown that molecular classification can aid in the treatment of patients with prostate cancer (Behrendt et al., 2014). At present, there are numerous molecular markers for the evaluation of the prognosis of prostate cancer, such as auxin, vascular endothelial growth factor, and transforming growth factor beta 1 , etc. These provide effective references for the clinical treatment and prognosis of prostate cancer (Rachner et al., 2014). 
In the present study, we analyzed the expression of S100P, CD147, and OCT4 in prostate cancer tissues with different TNM staging. Previous studies had confirmed that neither monoclonal nor polyclonal S100P antibodies resulted in detection of positive expression in prostate cancer (Nguyen et al., 2014). Xiang et al. (2014) also found no expression of S100P in prostate cancer by cDNA microarray. In our study, the positive rate for S100P expression in prostate cancer tissues was $18.52 \%$, which was significantly lower than in tissues from normal individuals and from those with prostate hyperplasia, consistent with other research (Liu et al., 2011). By analyzing different prostate cancer tissue TNM staging, we further demonstrated that $\mathrm{S} 100 \mathrm{P}$ had a positive rate of 0 in stage $\mathrm{V}$, significantly lower than that in stages I (37.50\%) and II $(35.71 \%)$. This result indicated there is a close relationship between prostate cancer stage and S100P expression. The S100P negative expression rate increased following stage increase, consistent with other published clinical research (Ricci et al., 2013). Previous studies showed that androgen receptor expression in cells overexpressing S100P increased significantly, and that there is a close relationship between androgen receptors and cell proliferation (Lee et al., 2011). Accordingly, Jiao et al. (2012) found that S100P expression promoted prostate cancer progression both in vitro and in vivo. Our study showed that the S100P expression positive rate in patients with distant metastasis was $4.00 \%$, significantly lower than that of patients without metastasis. In addition, many studies have shown that S100P expression has no significant correlation with prostate cancer differentiation degree (Crea et al., 2012); our results were consistent with these studies (Ricci et al., 2013).

CD147 is a type of cell adhesion molecule that is generally expressed at low levels in healthy people. It is primarily expressed in malignant tumors such as thyroid, breast, laryngeal squamous, and liver cancers and in cervical adenocarcinoma. It was initially used for the differential diagnosis of breast cancer. It was found that CD147 can also be expressed in prostate cancer and also had a certain value in evaluating the diagnosis and prognosis of patients with prostate cancer. In present study, we found that the CD147 expression positive rate in prostate cancer was $100.00 \%$, significantly higher than in normal and hyperplastic tissues. These results are comparable to the result presented by Ugolkov et al (2011). Our study also found that the majority of prostate cancer tissues were strongly positive for CD147 expression, accounting for $57.14 \%$. It has been shown that CD147 can enhance the synthesis and secretion of matrix metalloproteinases, which can promote cancer metastasis and invasion by damaging cell stromal elements and extracellular basement membrane (Li et al., 2010). In the present study, the CD147 positive expression rate in prostate cancer with distant metastasis was $100.00 \%$, demonstrating that the high expression CD147 rate has a close relationship with prostate cancer distant metastasis. However, the CD147 positive expression rate was found to have no relationship with the degree of differentiation of the cancer, which is consistent with a number of previously published results (Jham et al., 2012).

OCT4 is a type of POU transcription factor that is overexpressed in undifferentiated embryonal carcinoma, reproductive stem cells, and embryonic stem cells. It has a very important role in maintaining the self-renewal and pluripotency ability of embryonic stem cells. Several studies have found that OCT4 expression in the healthy population is low, whereas OCT4 expression is high in gallbladder carcinoma, pancreatic cancer, and liver cancer (Wang et al., 2012). Specially, its expression level is upregulated following clinical upstaging. In pancreatic cancer, OCT4 sensitivity was higher, but no significant correlation was found with the differentiation state of pancreatic cancer. Our research showed that the positive rate of OCT4 expression in prostate cancer was $77.38 \%$, markedly higher than that in normal and 
hyperplastic tissues. The OCT4 positive rate in prostate cancer stages III and V was 86.67 and $94.12 \%$, respectively, significantly higher than in stage I. This demonstrated that OCT4 has a close relationship with TNM staging, consistent with previously published research (Kregel et al., 2013). In addition, in line with a report by Li et al. (2013), we also found that OCT4 expression rates of tissues at different degrees of differentiation were similar, suggesting that OCT4 expression has no close relationship with prostate cancer differentiation.

In conclusion, $\mathrm{S} 100 \mathrm{P}$ was significantly downregulated in prostate cancer, whereas CD147 and OCT4 expression showed the opposite pattern. All factors demonstrated a close relationship with TNM staging and with distant metastasis in prostate cancer, but were less correlated with degree of differentiation. We did not analyze the relationship of their expression level and prognosis; this will be analyzed using a larger sample size in subsequent studies.

\section{REFERENCES}

Behrendt K, Nowicka E, Gawkowska-Suwinska M, Piewicki G, et al. (2014). Early closure of phase II prospective study on acute and late tolerance of hypofractionated radiotherapy in low-risk prostate cancer patients. Rep. Pract. Oncol. Radiother. 19: 337-342.

Crea F, Fornaro L, Bocci G, Sun L, et al. (2012). EZH2 inhibition: targeting the crossroad of tumor invasion and angiogenesis. Cancer Metastasis Rev. 31: 753-761.

Javanmard B, Hassanzadeh Haddad A, Yaghoobi M and Lotfi B (2014). Diode laser ablation of prostate and channel transure notthral resection of prostate in patients with prostate cancer and bladder outlet obstruction symptoms. Urol. J. 11: 1788-1792.

Jham BC, Costa NL, Silva JM, de Miranda AC, et al. (2012). Midkine expression in oral squamous cell carcinoma and leukoplakia. J. Oral Pathol. Med. 41: 21-26.

Jiao J, Hindoyan A, Wang S, Tran LM, et al. (2012). Identification of CD166 as a surface marker for enriching prostate stem/progenitor and cancer initiating cells. PLoS One 7: e42564.

Jones DR (2014). Measuring midkine: the utility of midkine as a biomarker in cancer and other diseases. Br. J. Pharmacol. 171: 2925-2939.

Kregel S, Kiriluk KJ, Rosen AM, Cai Y, et al. (2013). Sox2 is an androgen receptor-repressed gene that promotes castration-resistant prostate cancer. PLoS One 8: e53701.

Lee EK, Cho H and Kim CW (2011). Proteomic analysis of cancer stem cells in human prostate cancer cells. Biochem. Biophys. Res. Commun. 412: 279-285.

Li K, Liu C, Zhou B, Bi L, et al. (2013). Role of EZH2 in the growth of prostate cancer stem cells isolated from LNCaP cells. Int. J. Mol. Sci. 14: 11981-11993.

Li T, Su Y, Mei Y, Leng Q, et al. (2010). ALDH1A1 is a marker for malignant prostate stem cells and predictor of prostate cancer patients' outcome. Lab. Invest. 90: 234-244.

Liu C, Kelnar K, Liu B, Chen X, et al. (2011). The microRNA miR-34a inhibits prostate cancer stem cells and metastasis by directly repressing CD44. Nat. Med. 17: 211-215.

Monma F, Hozumi Y, Ikematsu S, Kawaguchi M, et al. (2013). Expression of midkine in normal human skin, dermatitis and neoplasms: association with differentiation of keratinocytes. J. Dermatol. 40: 980-986.

Nguyen PL, Aizer A, Assimos DG, D'Amico AV, et al. (2014). ACR Appropriateness Criteria (R) definitive externalbeam irradiation in stage T1 and T2 prostate cancer. Am. J. Clin. Oncol. 37: 278-288.

Rachner TD, Thiele S, Göbel A, Browne A, et al. (2014). High serum levels of Dickkopf-1 are associated with a poor prognosis in prostate cancer patients. BMC Cancer 14: 649 .

Ricci E, Mattei E, Dumontet C, Eaton CL, et al. (2013). Increased expression of putative cancer stem cell markers in the bone marrow of prostate cancer patients is associated with bone metastasis progression. Prostate 73: 1738-1746.

Tong SJ, Liu J, Wang X and Qu LX (2014). MicroRNA-181 promotes prostate cancer cell proliferation by regulating DAX-1 expression. Exp. Ther. Med. 8: 1296-1300.

Ugolkov AV, Eisengart LJ, Luan C and Yang XJ (2011). Expression analysis of putative stem cell markers in human benign and malignant prostate. Prostate 71: 18-25.

Vasiljevic N, Ahmad AS, Thorat MA, Fisher G, et al. (2014). DNA methylation gene-based models indicating independent poor outcome in prostate cancer. BMC Cancer 14: 655. 
Wang G, Wang Z, Sarkar FH and Wei W (2012). Targeting prostate cancer stem cells for cancer therapy. Discov. Med. 13: 135-142.

Xiang YZ, Jiang SB, Zhao J, Xiong H, et al. (2014). Racial disparities in the association between diabetes mellitusassociated polymorphic locus rs4430796 of the HNF1betagene and prostate cancer: a systematic review and metaanalysis. Genet. Mol. Res. 13: 6582-6592. 